\title{
EXPERIMENTAL AND NUMERICAL ANALYSES OF A 3D-PRINTED TITANIUM TRABECULAR DENTAL IMPLANT
}

\author{
Luboš ŘEhOuneK*, Aleš JíRA \\ Czech Technical University in Prague, Faculty of Civil Engineering, Thákurova 7166 29, Prague, Czech \\ Republic \\ * corresponding author: lubos.rehounek@fsv.cvut.cz
}

Abstract. The main focus of this paper is to investigate and describe a novel biomaterial structure. The trabecular structure has only recently been recognized as a viable alternative for prostheses and implants and seems to have very promising biocompatibility and mechanical properties. The 3D printing technique was used to create test specimens. These specimens were then tested by nanoindentation and tensile and compression tests. A numerical model was created and curve-fitted to represent the mechanical behavior of the trabecular structure. A significant reduction in the values of Young's modulus $E$ was observed. The values of $E$ for conventional implant materials are approximately 110-120 GPa and the trabecular structure reached a value just below $1 \mathrm{GPa}$. The next effort will be to apply the model onto a real implant. It is the "four leaf clover" implant variant by authors F. Denk Jr., A. Jíra and F. Denk Sr.

KEYWORDS: biomechanics; dental implant; trabecular; numerical model; 3D printing.

\section{INTRODUCTION}

The methods of manufacturing are constantly evolving and the whole idea of implants is growing rapidly [1]. With the technology we have at our disposal today, new implants become more and more available. Whereas before only people of privileged position could afford to undergo this kind of treatment, now it is a widely available and also suggested option for nearly everybody who suffers from damage related to their bone tissue [2]. We would like to present a comparison to confirm this fact. In the year 2008, searching the term "implant" in the PubMed database gave a total of 46575 papers, with 9768 of them being identified to the term "dental implant" (21\%). Now, at the time of writing this article in 2017, the same search extracted 100634 papers when searching "implant" and 38357 papers for "dental implant" (38\%). The total value has more than doubled and the number of papers written specifically on dental implants has quadrupled. This simple comparison shows us how much implants (dental implants in particular) have gained in popularity and attention over the course of mere 9 years.

Dental implants have advanced tremendously, evolving from primitive hammered-down pieces of bone into fine, precisely shaped metal products [2]. Yet, they still have one common denominator, and that is the bad interconnection between the implant and the bone caused by the difference of material properties at the interface of individual dental materials [3. Trabecular structure is a future prospect and a potential solution to the problem of both the bad interconnection and material difference at the interface of materials. If proven to be satisfying in medical, mechanical and economic regards, trabecular structure could improve upon the former variants.
Moreover, the structure promotes the formation of blood vessels in the newly formed bone tissue because of its porosity. Studies performed in-vitro (outside of the organism) show that this structure promotes osseointegration [4]. It has been reported that the integration of a trabecular implant and vascularization can occur as fast as in two weeks $[5$.

\subsection{Aim OF THE RESEARCH}

The complete mechanical analysis of the trabecular structure demands mechanical testing as well as a numerical model. For the purpose of this experiment, a new variant of the trabecular structure has been created. With sufficient data provided by mechanical tests, it is possible to develop a numerical model including the nonlinear behavior required to describe the trabecular structure.

We performed nanoindentation and uniaxial tensile and compression tests. The nanoindentation method was used mainly to determine the material properties on the micro level and compare them with the values given by the manufacturer. The global mechanical tests served a greater purpose - determining the values of Young's modulus and obtaining the stress-strain diagrams used as a baseline for the development of the numerical model. This model will be used in near future to determine the mechanical behavior of a more complex implant that incorporates both the homogeneous and trabecular structures (Fig. 2).

\section{Trabecular structure}

The trabecular structure is a structure that resembles the morphology of natural cancellous bone. The main reason behind creating complex structures, such as the trabecular structure, is simple - conventional implants are still far too apart from being in alignment with the 


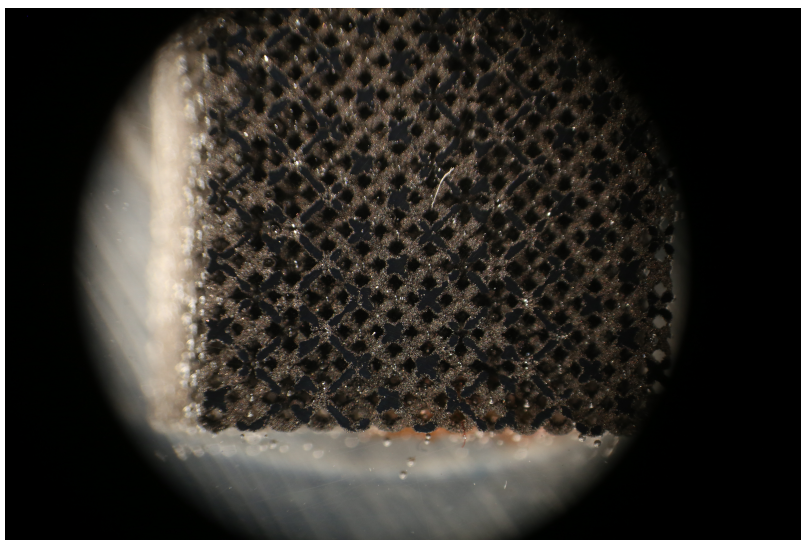

FIGURE 1. A micrograph of a polished cross-section of the 3D-printed trabecular structure. Note the individual levels of beams embedded in the 3D trabecular matrix. The edge of the specimen measures $14 \mathrm{~mm}$.

material properties of human tissues. Conventional implants have well-proven long-term success rates [6, 7, but are still prone to failure in the early stages of the bonding process, as geometry and surface properties play an important role in osseointegration [1].

The trabecular structure aims to lower the global modulus of the final product by reducing the volume of material (Fig. 1) within its body. Its morphology (Fig. 3) tries to imitate human cancellous bone in this manner. The shape of the structure is also beneficial in regard to osseointegration, as it is porous and allows for bone ingrowth 8,9 and provides a more "rough" surface (from the macro point of view).

This experiment aims to describe this particular trabecular structure, but there can be many variants of its shape. Osseointegration is expected to significantly vary with different magnitudes of pores and the shape of the trabecular cells as the character of bone ingrowth will be different. Mechanical properties also vary with different widths of individual beams, their density inside the trabecular matrix and similar parameters. Observing and manipulating these parameters is important for optimal results, but it is not the aim of this research at this time.

There are generally 3 main attributes in which the trabecular structure aims to improve upon its former variants - increasing osseointegration, lowering the modulus and reducing stress-shielding.

\subsection{OSSEOINTEGRATION}

The geometry of the trabecular structure forms the bearing scaffold for the ingrowth of bone cells into the implant [8, 9. This interconnection is beneficial for long term stability at the implantation site [10. With conventional implants, bone cells are only able to grow onto the surface of the implant. With the trabecular structure, however, the cells can grow inside and create an interconnected material comprising both bone and metal bonded together (Fig. 10p.

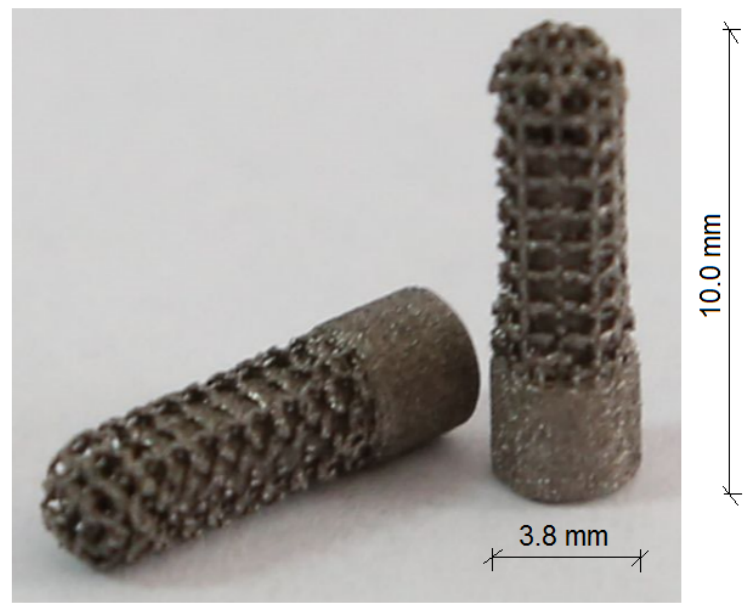

Figure 2. Whole implant body of the "four leaf clover implant", consisting of both the trabecular and homogeneous structure. Implant has been patented in Czech Republic [14].

Surface roughness has been proven to play an important role in the bonding process [11-13. The greater the surface area of the implant, the greater the interlocking between the implant surface and bone. Therefore, the trabecular structure has a potential of a much better bonding and creating a fluent material transition region as its morphology resembles a material with high surface roughness (Fig. 2). However, due to the lack of experimental data, this assumption is still to be proven.

\subsection{Elastic PROPERTIES}

The second attribute is Young's modulus. While the reduced modulus of the material on the micro level (modulus of the bulk material, not of the whole structure) remains unchanged, the global modulus of the whole structure is expected to be dramatically reduced since the cross-section of the whole body of the implant is also reduced. This reduction helps to smoothen the material transition region, where unwanted stresses often cause large deformations, leading to an implant loosening. As shown in Fig. 2, the trabecular outer shell provides an interconnecting layer between the bone and the stem of the implant.

\subsection{StREss ShiELDing}

Stress-shielding is another factor which is governed by the values of the moduli of different dental materials. It is an unwanted factor that represents uneven distribution of stress between the implant, peri-implant area and bone [15. Since the modulus of the bone is much lower than that of the implant (approximately 20-30 GPa [16] for bone and 90-110 GPa for conventional titanium implants [17]), stress is transferred into the implant, leaving the bone without sufficient stimulus. By replacing bone with an implant, which has a higher value of the modulus, we modify the stress distribution that occurs under load. 


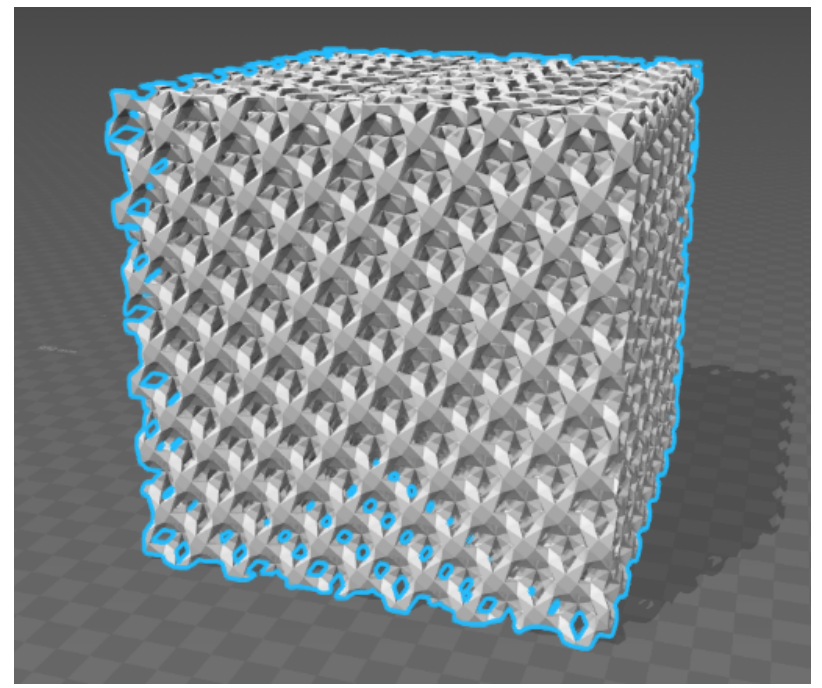

Figure 3. A 3D STL model of trabecular structure used to create metal test specimen by means of $3 \mathrm{D}$ printing.

At this point, the Wolff's law plays an important role. Naturally, the bone is provided by an evenly redistributed stress that provides all of its areas with sufficient load to maintain its mass, but after implantation, the bone around the implant starts to remodel itself and deteriorate [18]. When the bone becomes overly porous, it is no longer able to hold the implant in position, making it eventually slip out, resulting in a failure of the implant.

\section{3D PRINTING}

\subsection{D PRINTING TECHNOLOGY}

Conventional machining does not allow to create more advanced and complex geometric structures. The trabecular structure comprises beams of equal length embedded into a 3D matrix, making 3D printing potentially the only technology available to produce it. The first step in creating a 3D-printed product is creating a 3D-model. This part is usually done using a computer-aided design (CAD) environment. Upon its completion, the STL model file (Fig. 3) is divided into thin cross sections [8, 9] and sent to the 3D printer to be processed. Up to this point, the process is similar to the common layer-by-layer 3D-printing of plastic.

What differentiates the process of printing metals (selective laser sintering) from the standard technology is using a laser beam to melt down a layer of metal powder. During each cycle, the coater applies a thin layer of powder, which is processed by a laser at a pre-set melting point in a pre-determined order. This process solidifies the loose powder into a 3D-layered object.

\subsection{PRODUCTION FLAWS}

Despite the fact that the trabecular structure has many benefits, it also has its flaws. While observing the quality of the 3D-printed specimens, many
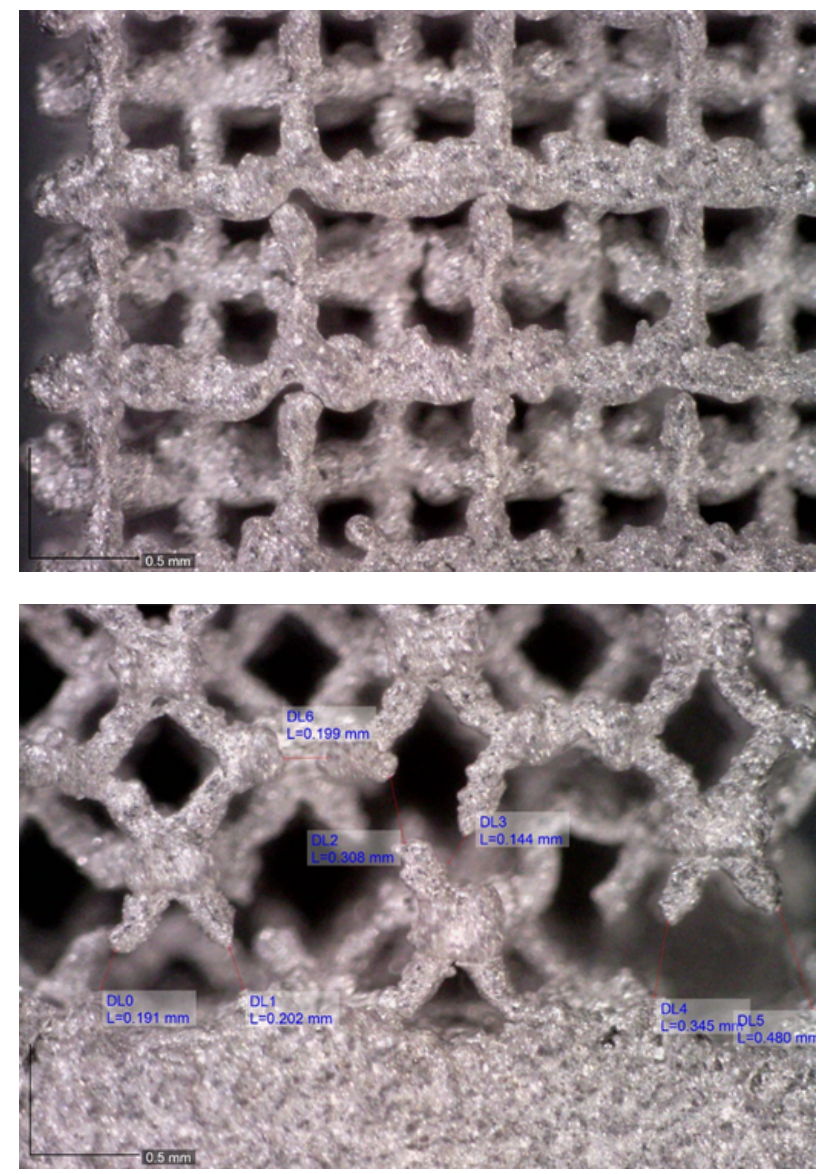

Figure 4. Production imperfections at the interface of the homogeneous and trabecular cross-sections of a tensile test specimen. Note the faulty intersections of the beams as well as the disconnection of particular beams and varying width. Associated text denotes the length of individual disconnections.

geometrical imperfections associated with the printing technology were discovered. These imperfections can hardly be incorporated into the model as their occurence seems to be purely random. The imperfections are shown in Fig. 4. They are, rather than a bad attribute of the structure itself, caused by the production process of the 3D printing. They form probably during the phase in which the machine applies a layer of powder and subsequently melts it by the laser beam. We expect these geometrical imperfections to be caused either by improper tracing of the laser beam, faulty metal powder dosage, or a combination of both. While the trabecular structure brings many benefits to the field of implantology, the technology of 3D printing still has to improve in order to provide stable, homogeneous outcomes and dependable products.

\section{Mechanichl tests}

\subsection{Test specimens}

A total of 12 specimens were created for the purpose of global mechanical tests - 3 for tensile and 9 for compression tests (as the compression failure charac- 

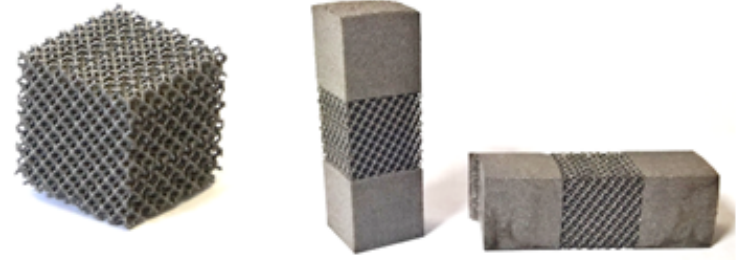

FiguRE 5. Trabecular structure specimens for global mechanical tests. Compression test specimen, dimensions $14 \times 14 \times 14 \mathrm{~mm}$ (left) and tensile test specimen, dimensions $14 \times 14 \times 42 \mathrm{~mm}$ (right).

teristics are unclear and are expected to be much more heterogeneous). Two other specimens were created to investigate the micromechanical properties by means of nanoindentation. The trabecular specimens were $3 \mathrm{D}$ printed using the M2 Cusing machine from the Concept Laser Company. The manufacturing of the specimens has been done in cooperation with ProSpon spol.s.r. o. A specialized input medium Rematitan CL was used for the printing of the test specimens. It is a $\mathrm{Ti}-6 \mathrm{Al}-4 \mathrm{~V}$ titanium alloy powder provided by the medical technology manufacturer Dentaurum.

For the purpose of mechanical tests, we used the 3D Dode-Thick [MSG] structures with dimensions of $14 \times 14 \times 14 \mathrm{~mm}$ (a cube for the compression test, Fig. 5 left) and $14 \times 14 \times 42 \mathrm{~mm}$ (a block for the tensile test, Fig. 5 right). The tensile test specimen had a $14 \mathrm{~mm}$ trabecular middle section and end portions of homogeneous volume for ensuring a better anchor in the MTS Alliance RT-30 machine. The inner dimensions of the structure are as follows - the axial distance between individual beams (trabecular cell width/depth) is between $0.86-0.90 \mathrm{~mm}$ and the beam width is usually between $0.10-0.18 \mathrm{~mm}$, in extreme inhomogeneous areas even up to $0.32 \mathrm{~mm}$.

The material used to create the specimens is a powder based on the Ti-6Al-4V titanium alloy. This alloy has been chosen because it is already approved for use as a biomaterial and has a long, well-documented history of success rates in clinical dentistry and is still widely used by implantologists all around the world.

\subsection{NANOINDENTATION}

The nanoindentation tests were made considering a reduced modulus of elasticity, hardness and contact depth and using the Oliver \& Pharr method [19]. The micromechanical analysis was performed using the CSM Instruments nanoindenter equipped with a Berkovich indenter tip. An image of a typical indentation matrix is shown in Fig. 6.

The two nanoindentation specimens were tested by a total of 7 indent matrices - three were situated in the first specimen and four in the second specimen. Their indentation curves are shown in Fig. 7. The mechanical properties obtained by nanoindentation are listed in Tab. 1 .

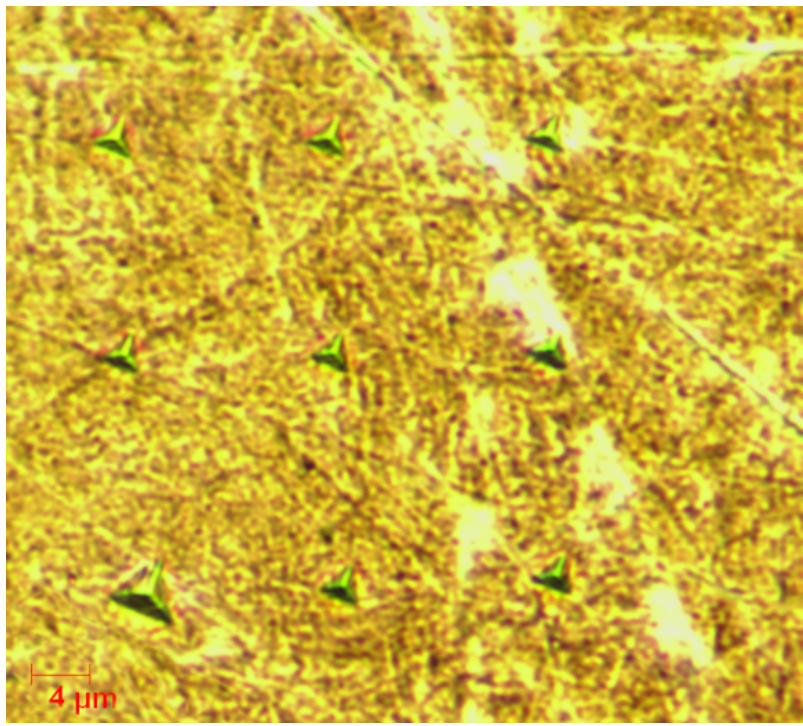

Figure 6 . A typical $3 \times 3$ indentation matrix with visible plastic deformation in the beams of the alloy. Maximum available resolution provided by the indenter microscope.

It is very important to distinguish between the results obtained by nanoindentation and global mechanical tests, especially when considering the trabecular structure. Nanoindentation represents the mechanical properties of the material on the micro level and global mechanical tests describe the properties of the whole tested specimen. While these two properties might be somewhat similar when testing a homogeneous specimen, it is vital not to mix them up when considering trabecular structure, where the values can vary as much as 100 times (Tab. 1 and Tab. 2).

All results obtained by nanoindentation (Tab. 1) show that the values of reduced modulus $E_{\mathrm{r}}$ (comparable to the modulus of the bulk material) fall in the range of 118-131 GPa. Considering the material list provided by the manufacturer, where the value of $E$ is $115 \mathrm{GPa}$, we can, with fair certainty, say that these values correspond. These material characteristics are also comparable to the conventional, machined Ti-6Al-4V implant material [17.

Nanoindentation tests have proven that the 3Dprinted material can be fully compared to conventionally created structures as the material properties given by the manufacturer of the metal powder correspond with the properties obtained on the micro level. The micromechanical analysis has also proven the values of properties to be dependent on the depth of the indent (or the size of the loading force).

\subsection{Global mechanical tests}

To investigate the global mechanical properties, we conducted compression and tensile tests. Global mechanical tests were performed using the MTS Alliance RT-30 machine. The compression test specimen did not require any further modifications, but the tensile test specimens had a $14 \mathrm{~mm}$ trabecular middle section 


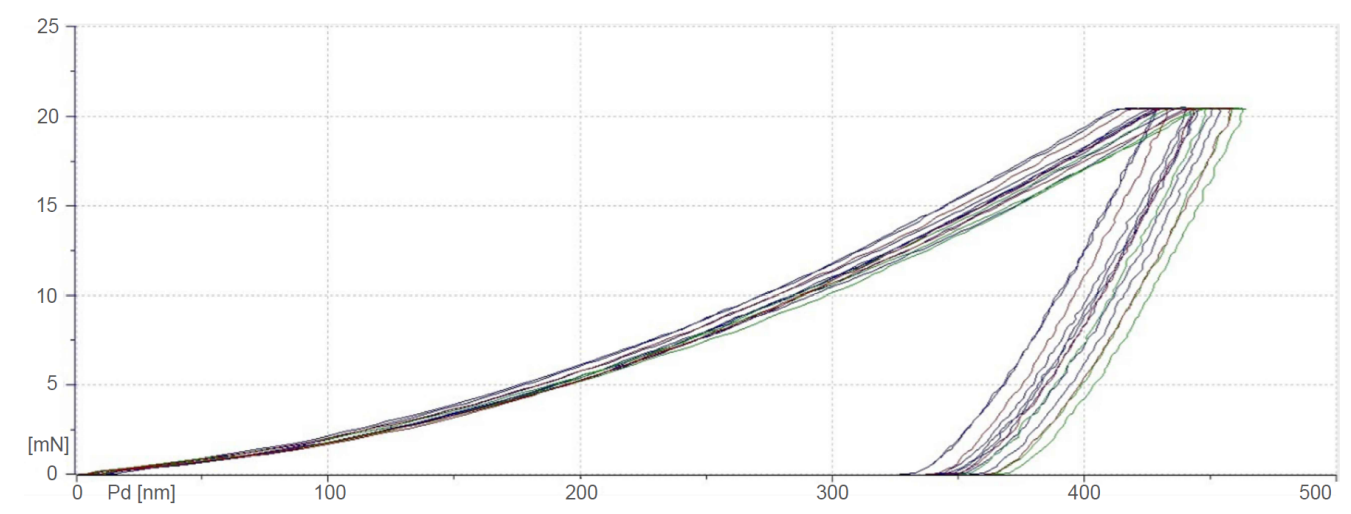

Figure 7. Three sets of indentation curves obtained from indentation of the first specimen.

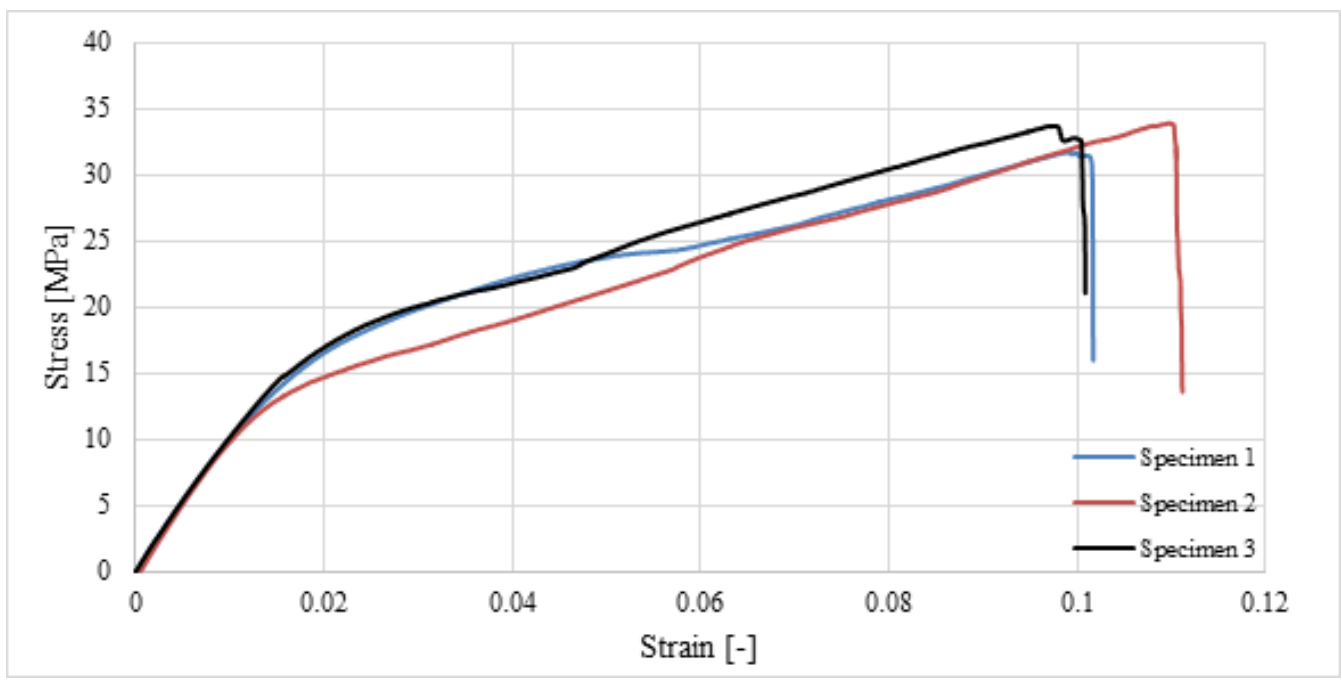

Figure 8. Stress-strain relation diagram of the tensile test specimens.

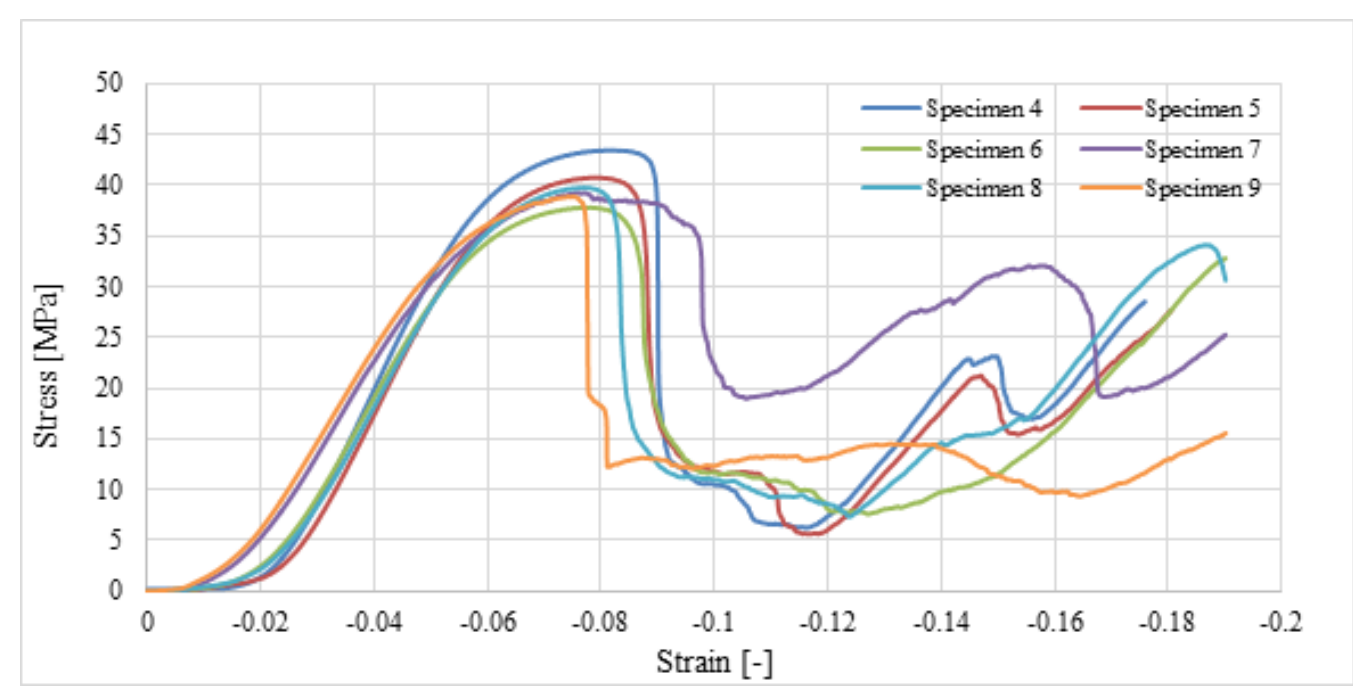

FiguRE 9. Stress-strain relation diagram of the compression test specimens. Note the very heterogeneous nature of compressive deformation beyond the failure of the specimen. This fact can be attributed to the individual layers of the trabecular structure locking into one another in a random fashion. 
and end portions of homogeneous volume for ensuring a better anchor in the MTS Alliance RT-30 machine (Fig. 5). The specimens were tested beyond the point of failure. Then, we calculated the values of the modulus $E$ by using the least squares method using the linear parts of the stress-strain diagrams of the curves shown in Fig. 8 and Fig. 9

The Young's modulus has been calculated from the linear parts of the stress-strain diagrams as a tangent. Only linear parts of the curves were considered as Young's modulus cannot be determined this way in the range of plasticity. For strain, these values range approximately from 0.00 to 0.15 for the tensile test and from -0.025 to -0.05 for the compression test. These values represent properties of the whole trabecular specimen, contrary to the nanoindentation experiment, where obtained values represent only the material characteristics at the micro level.

Knowing the exact dimensions of the specimens and the time-dependent indenter force, we were able to recalculate the force into stress by using a simple formula:

$$
\sigma=\frac{F}{A}
$$

where $\sigma$ represents stress, $F$ represents the timedependent load applied by the machine and $A$ represents the cross-section area of the specimen. The dimensions of the tensile test specimen are $14 \times$ $14 \times 42 \mathrm{~mm}$ (Fig. 5, so the cross-section area equals $196 \mathrm{~mm}^{2}$. The strain has been calculated using another simple formula:

$$
\varepsilon=\frac{D}{L}
$$

where $\varepsilon$ represents strain, $D$ represents the longitudinal displacement (extension) and $L$ represents the total length of the trabecular middle portion $(14 \mathrm{~mm})$.

If the structure had its beam structure and width experimentally manipulated in order to match the modulus of the human bone, it would not necessarily be prolific as more data and in-vivo experiments are still needed. Porosity, beam width and surface morphology are important in order to fully osseointegrate the implant and induce bone ingrowth, which is one of the main potentials of this structure. Therefore, observing the results from in-vivo tests can be a far more productive approach than exactly matching the properties of the structure and the human bone. Since bone ingrowth is one of the major benefits of the trabecular structure, its maximizing will have priority over an exact value of the modulus.

\section{IN-VIVO TESTS}

We have conducted in-vivo tests of the trabecular implant specimens. The tests were carries out on laboratory pigs. The whole purpose of the in-vivo tests was to observe the healing process and ultimately determine the osseointegration of the trabecular implant specimens after 6 months following implantation. The
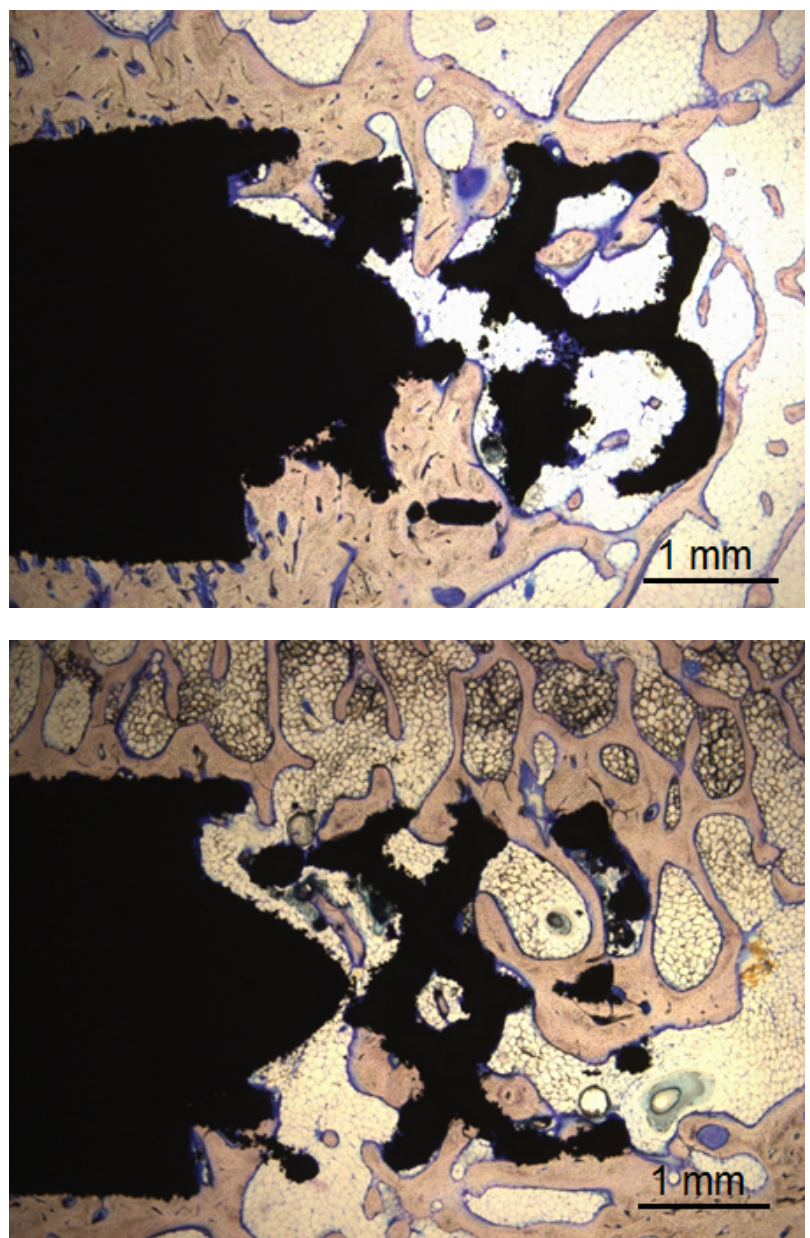

Figure 10. Two micrographs of longitudinal sections of extracted trabecular specimens. The samples were colored using Giems solution azur-eosin-methylene blue. White and light yellow color represents fibrous tissue, orange represents beams of newly formed bone tissue and black color represents the trabecular implant. Specimen n.1 (top) and specimen n.2 (bottom)

implants were placed in the proximal area of the femur of pigs. There was a total of 34 implants placed in 4 pigs. Six months after the operation, the bones were extracted and tissue bars $3 \mathrm{~cm}$ long and $1-3 \mathrm{~cm}$ wide were made in order to examine the individual bonding abilities of various implant shapes. Subsequently, the samples were cut in half and their surface was prepared in a similar fashion as the aforementioned nanoindentation specimens.

Conducted in-vivo tests on pigs show that the living tissue is able to grow inside the trabecular structure and form new beams of bone directly in contact with the beams of the trabecular structure (Fig. 10). However, full osseointegration was not achieved. The percentage of bone-implant contact after 6 months from implantation is, however, still worse when compared to the conventional homogeneous implants. Our opinion is that more tests will definitely be needed as a single in-vivo test cannot provide a dependable 
result and the trabecular structure might even need slightly different techniques of implantation and healing periods.

To quickly evaluate the outcome of these in-vivo tests, it was discovered that the bone-implant contact of the trabecular structure was inferior to that of the conventional homogeneous implants. However, it was still better than the remaining 2 groups of experimental implants. Bone-implant contact is a major indicator of osseointegration, but more tests will be needed in order to gain more data and evaluate the experiments in different conditions and with different parameters.

\section{NumeriCAL MODEL}

\subsection{InTRODUCTION OF THE MODEL}

The purpose of the model can be twofold - to apply the material characteristics to the trabecular structure and fully represent its behavior or to apply these characteristics to a homogeneous structure that will then represent the trabecular structure as a homogeneous material with the mechanical properties of the trabecular structure. The first approach is able to directly characterize the behavior of the trabecular structure. However, considering the complexity of the trabecular structure and the many problems that had arisen during the process of the numerical analysis, it is also possible that the whole process will be much more time-consuming. Because of that, we considered the application of the trabecular properties onto a homogeneous structure a viable and functional alternative.

With the numerical model at hand, it will be possible to evaluate the stress distribution throughout implants of different shapes and structures. This comparison can then be used to manipulate the shape of the trabecular structure, structural portions of implants, width of the beams and their distance. Such comparison will lead to implant shapes that will have smoother stress distribution throughout their bodies, reduced stress-shielding and unwanted stress concentrations.

\subsection{Methodology}

Our approach in the development of the model was to try to imitate the process of the tensile mechanical test to the best of our abilities. For this purpose, we used the Ansys Workbench 16.2. software for a finite element method analysis. Because we had the test data from the MTS Alliance RT-30 test machine, we had full knowledge of the force-displacement relations.

The machine was recording the loading force and longitudinal displacement in $0.1 \mathrm{~s}$ long time steps. The whole experiment took about 430-470 seconds till failure of the specimen for all three specimens. The amount of data can then be considered sufficient as more than 4000 individual force-displacement points were recorded for each specimen. This data was then

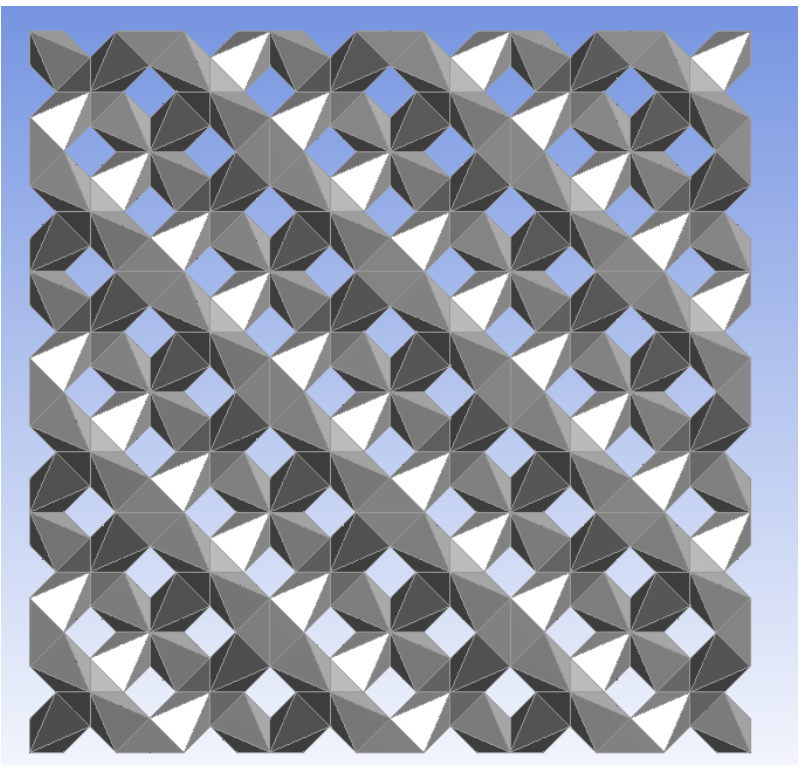

Figure 11. The new 3D model created in Design Modeler of Ansys Workbench, comprising of three basic elements per edge.

used to calculate stress and strain and the displacement was also utilized in the numerical analysis in the loading program.

The displacement provided by the loading machine was recorded for the absolute time frame $(430-470$ seconds for all specimens) and subsequently used as a displacement load in the numerical loading program to simulate the exact process of the tensile test.

\subsection{Geometrical MODEL}

We decided that performing the analyses on a smaller model will be a better, more time-conserving approach. We swapped the model shown in Fig. 3 for a smaller model shown in Fig. 11. Since the only other model structure available was the basic structure of the single element, we used it to create a model consisting of these basic elements. To scale it down in comparison to the default model, we used three of these elements per edge of the imaginary circumscribing cube of the structure, as opposed to seven elements per edge in the former STL model used to produce the test specimens.

\subsection{Meshing And LOAD PROGRAm}

With the geometry prepared, the next step in the numerical analysis was to create a proper mesh. While performing this task, we ran a couple of simple computations with elementary loads. The purpose of these computations was to determine how much does the size of the elements and their quantity influence the computation time. As a result of these mini-computations, we chose to incorporate a mesh that covers every single beam of the structure by a minimum of three elements. This quantity seems to be small enough in regard to computation time and also great enough to provide the necessary precision of the solution. We also tried variants with more elements per the width of a beam, 


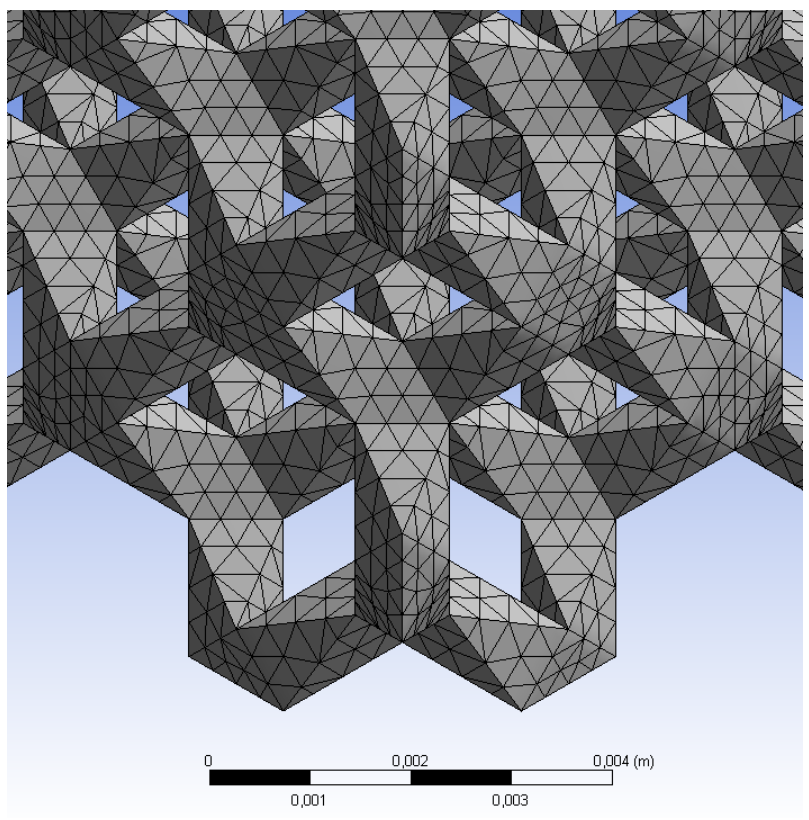

Figure 12. The FEM mesh of the trabecular structure generated by the Ansys Workbench software. The mesh elements are quadratic tetrahedrons.

but have concluded that computation times were increasing so quickly that such an environment would have been absolutely unsuitable for the curve-fitting process.

The provided load has been implemented in the form of tabular data as displacement of the specimen number 3 obtained from the tensile test. For this particular specimen, the test took 420 seconds until failure. We have decided to abbreviate this process and include only every tenth value of displacement, shortening the process into 42 load values. This reduction has been done to minimize the amount of input data as the computer could not handle so many load steps (effectively freezing for several minutes by merely accessing the data). Knowing that the displacement is a near-linear curve, this reduction of load points did not interfere with the precision of the analysis.

\subsection{Curve-Fitting}

The material model used for the curve-fitting process was the Ti-6Al-4V material provided by the Ansys Workbench Explicit Dynamics material library. It is a material model with a bilinear stress-strain diagram with isotropic hardening. The stress tensor is separated as a uniform hydrostatic stress and completed with a deviatoric stress tensor via using the equations of state bound with local energy. The first equation of state for compression $(\mu>0)$ is as follows:

$$
p=A_{1} \mu+A_{2} \mu^{2}+A_{3} \mu^{3}+\left(B_{0}+B_{1} \mu\right) p_{0} e
$$

and the second for tension $(\mu<0)$ is:

$$
p=T_{1} \mu+T_{2} \mu^{2}+B_{0} p_{0} e,
$$

where

$$
\mu=\text { compression }=\rho /\left(\rho_{0}-1\right) .
$$

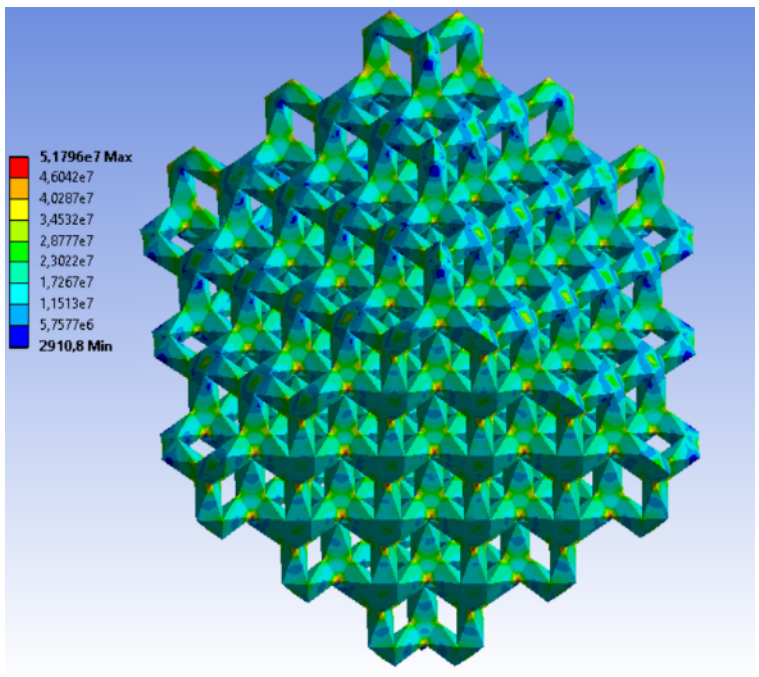

Figure 13. The model as loaded with displacement load, isolines show equivalent (von-Mises) stress distribution in $\mathrm{Pa}$.

where $\rho_{0}$ is density at zero stress, e is inner energy per unit of mass and $A_{1}, A_{2}, A_{3}, B_{0}, B_{1}, T_{2}$ and $T_{2}$ are material constants (either in Pa or dimensionless).

By experimentally manipulating the values of additional properties, we were able to fit the material properties of the newly introduced material so as to imitate the tensile test. The final values, which have been manipulated during the curve-fitting process, have been saved and recorded for further efforts.

Since there is only a single load, the analysis is not complex and does not require additional timeseparated intervals (unlike, for example, a gasket assembly analysis with tightening of bolts, subsequent thermal load etc.). It was performed in one computation step. The stress-strain diagram, which we were trying to curve-fit, is the specimen number 3 shown in Fig. 8 The convergence of the solution is attained by the Newton-Raphson method.

The two main properties that we were changing throughout the whole process were the values of Young's modulus $E$ (the slope of the curve up until the range of yield strength) and tangent modulus $E_{\mathrm{t}}$. Tangent modulus is a material property provided for non-linear analyses. It represents the slope of the curve in the range between the yield and ultimate strength. The comparison of the experimental data and the final solution is shown in Fig. 14.

\section{Conclusions}

\subsection{Results}

The mechanical tests have shown that by incorporating the trabecular structure, we are able to significantly reduce the modulus of the structure (Tab. 1] and Tab. 22. This reduction is beneficial as the effects of stress-shielding are reduced and the mechanical properties of the implant are more similar to the human bone. 


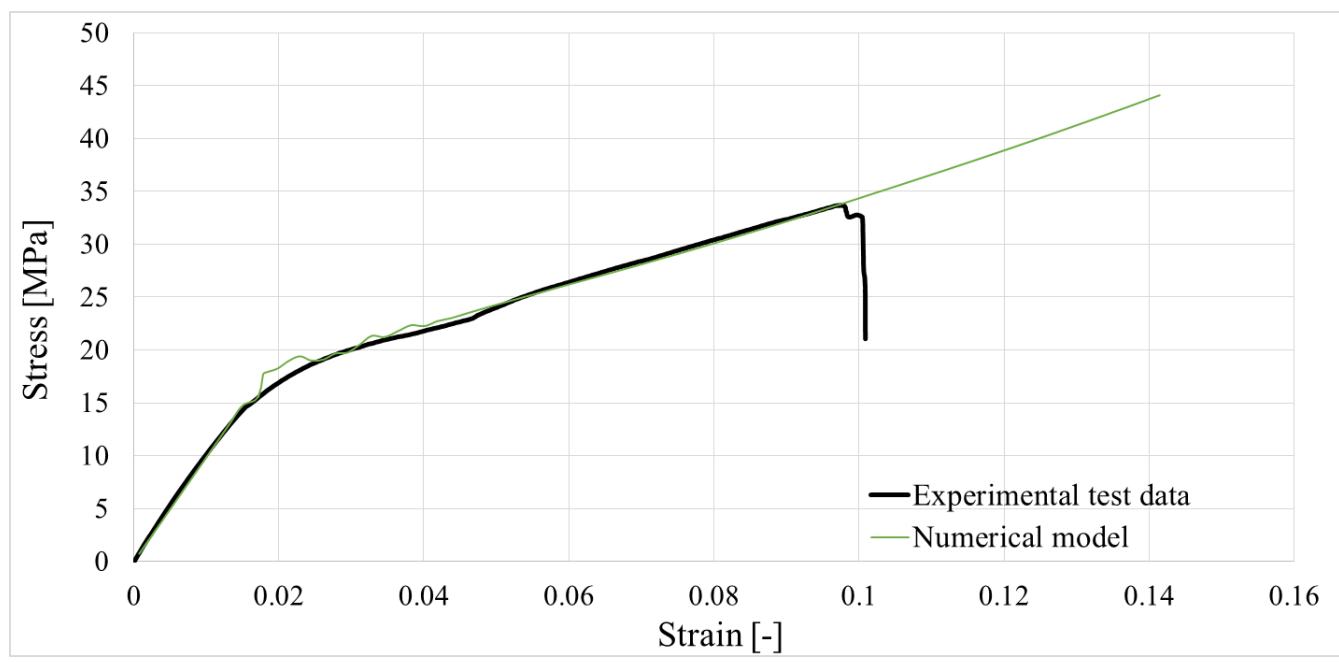

FiguRE 14. The final curve containing the final material properties (green) in comparison with the experimental test data (black). The complete result of the curve-fitting process. Unattained failure beyond the region of ultimate strength is acknowledged and will be improved upon in the future.
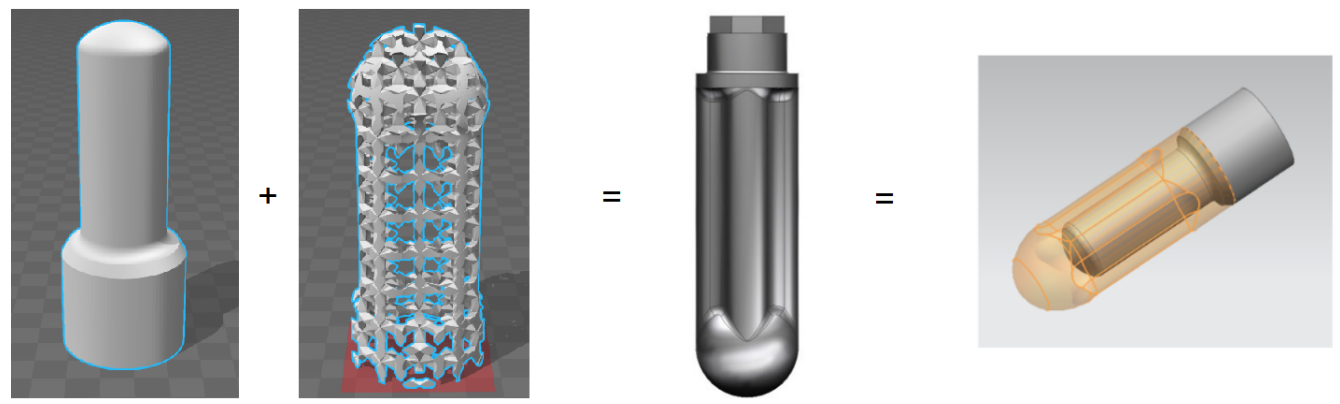

Figure 15. The "four leaf clover" implant variant, comprising of a cylindrical body and four rounded grooves encompassed in a hemisphere (in the middle) and the two potential approaches towards modelling the structure. Two STL files for direct characterization (left) and a model using the homogenized structure (right). The implant has been patented in Czech Republic [14].

\begin{tabular}{lccccccc}
\hline Property & \multicolumn{7}{c}{ Load cycle } \\
\cline { 2 - 8 } & 1 & 2 & 3 & 4 & 5 & 6 & 7 \\
\hline$H_{\text {it }}[\mathrm{MPa}]$ & 5191 & 5201 & 5188 & 5166 & 5191 & 5202 & 5191 \\
Std. dev. & 251 & 200 & 281 & 343 & 379 & 404 & 395 \\
\hline$E_{\mathrm{r}}[\mathrm{GPa}]$ & 131 & 122 & 119 & 118 & 117 & 117 & 118 \\
Std. dev. & 10 & 6 & 4 & 3 & 3 & 3 & 4 \\
\hline$H_{\mathrm{c}}[\mathrm{nm}]$ & 267 & 381 & 471 & 544 & 608 & 660 & 732 \\
Std. dev. & 7 & 8 & 13 & 22 & 25 & 31 & 45 \\
\hline
\end{tabular}

TABLE 1. Mean values of reduced modulus $E_{\mathrm{r}}$, hardness $H_{\mathrm{it}}$ and contact depth $H_{\mathrm{c}}$ in individual load cycles.

\begin{tabular}{lcccccccccc}
\hline \multicolumn{10}{c}{ Young's Modulus $E[\mathrm{MPa}]$} \\
\hline Specimen & 1 & 2 & 3 & 4 & 5 & 6 & 7 & 8 & 9 & Mean \\
\hline Tens. test & 964.7 & 975.9 & 982.2 & - & - & - & - & - & - & $\mathbf{9 7 4 . 3}$ \\
Comp. test & - & - & - & 1114.2 & 1080.6 & 947.2 & 818.8 & 999.6 & 803.8 & $\mathbf{9 6 0 . 7}$ \\
\hline
\end{tabular}

TABLE 2. Values of Young's modulus obtained by global mechanical analysis. 
The values of individual properties are dependent on the depth of the indent. From a contact depth of $H_{\mathrm{c}}=470 \mathrm{~nm}$ (corresponding force of $20 \mathrm{mN}$ ), the trend of $E_{\mathrm{r}}$ and $H_{\mathrm{it}}$ is constant. We can therefore assume the loading force of $20 \mathrm{mN}$ as a basic value. The corresponding properties of this force are reduced modulus $E_{\mathrm{r}}=118 \mathrm{GPa}$ and hardness $H_{\mathrm{it}}=5187 \mathrm{MPa}$. The indentation hardness $H_{\text {it }}$ is calculated from the indenter force, divided by the projected area of the indenter in contact with the specimen at maximum load.

As shown in Tab. 1 and Tab. 2, the values of modulus vary dramatically. By introducing the trabecular structure, the modulus of the structure was lowered more than 100 times. This outcome is purely experimental and can vary amongst different structures with various dimensions. However, we believe that the values of modulus do not necessarily need to exactly match human bone as many more aspects, such as size of the pores and their shape come into consideration when evaluating biocompatibility.

The potential biomechanical benefits of the trabecular structure are still to be proven as there is not enough data and no documented history of success rates. Trabecular structure allows for both bone ongrowth and ingrowth, as opposed to the homogeneous implants which only allow the bone to grow on the surface of the implant. In-vivo tests have shown that bone cells are able to grow into the beams of the structure, creating better potential for osseointegration and interlocking of the implant and the surrounding bone.

\subsection{Future prospects}

The curve-fitting process was completed successfully with the exception of attaining failure beyond the range of ultimate strength. This is true due to the fact that the Ansys Workbench software is unable to provide solution including the failure of the model without using advanced methods. The approach towards the failure of the model can be twofold - either by implementing additional APDL commands or using the xFEM fracture mechanics. One of these approaches will be used in the near future to improve the model so as to attain the failure.

The aforementioned novel implant type "four leaf clover" was introduced because it will be the implant used for further applications of the numerical model. The variant shown in Fig. 15 is a conventionally machined implant, but our further effort will be to create a model that will combine both the homogeneous and the trabecular structure. This will be done by combining a homogeneous, machined stem with a trabecular, 3D-printed outer shell. The implant has been patented in the Czech Republic [14.

This conjunction will ultimately be the purpose of the model as a detailed analysis will be needed to determine the stress distribution and character of failure of an implant that combines multiple materials. The main goal will be to create a model consisting of both the homogeneous and the trabecular structure. That way it is possible to observe the full mechanical behaviour of the morphologically complex structure as it truly represents the real implant. The STL files needed to create the model and merge the two materials together are already available to us (Fig. 15).

With the curve-fitted material properties at hand, the next task will be to apply these properties onto an implant incorporating both the trabecular and homogeneous structures (Fig. 15 and observe its mechanical behavior. The analysis of this merged implant will be the final goal of this research.

\section{ACKNOWLEDGEMENTS}

The financial support by the Faculty of Civil Engineering, Czech Technical University in Prague (SGS project No. SGS17/168/OHK1/3T/11) is gratefully acknowledged. We would also like to extend gratitude to IAPG AS CR, v. v.i. for providing us with the environment for in-vivo experiments.

\section{REFERENCES}

[1] L. Le Guéhennec, A. Soueidan, P. Layrolle, Y. Amouriq. Surface treatments of titanium dental implants for rapid osseointegration. Dental Materials 23(7):844-854, 2007. DOI:10.1016/j.dental.2006.06.025.

[2] C. M. Abraham. A brief historical perspective on dental implants, their surface coatings and treatments. The open dentistry journal 8:50-5, 2014. DOI:10.2174/1874210601408010050

[3] B. V. Krishna, S. Bose, A. Bandyopadhyay. Low stiffness porous Ti structures for load-bearing implants. Acta Biomaterialia 3(6):997-1006, 2007. DOI:10.1016/j.actbio.2007.03.008

[4] F. Benazzo, L. Botta, M. F. Scaffino, et al. Trabecular titanium can induce in vitro osteogenic differentiation of human adipose derived stem cells without osteogenic factors. Journal of Biomedical Materials Research - Part A 102(7):2061-2071, 2014. DOI:10.1002/jbm.a.34875.

[5] J. D. Bobyn, K. K. Toh, S. A. Hacking, et al. Tissue response to porous tantalum acetabular cups: A canine model. Journal of Arthroplasty 14(3):347-354, 1999. DOI:10.1016/S0883-5403(99)90062-1

[6] T. Hasegawa, S. Kawabata, D. Takeda, et al. Survival of Brånemark System Mk III implants and analysis of risk factors associated with implant failure.

International journal of oral and maxillofacial surgery 46(2):267-273, 2016. DOI:10.1016/j.ijom.2016.10.014.

[7] F. De Angelis, P. Papi, F. Mencio, et al. Implant survival and success rates in patients with risk factors: results from a long-term retrospective study with a 10 to 18 years follow-up. European review for medical and pharmacological sciences 21(3):433-437, 2017.

[8] F. E. Wiria, J. Y. M. Shyan, P. N. Lim, et al. Printing of Titanium implant prototype. Materials and Design 31(SUPPL. 1):S101-S105, 2010. DOI:10.1016/j.matdes.2009.12.050 
[9] A. El-Hajje, E. C. Kolos, J. K. Wang, et al. Physical and mechanical characterisation of 3D-printed porous titanium for biomedical applications. Journal of Materials Science: Materials in Medicine 25(11):24712480, 2014. DOI:10.1007/s10856-014-5277-2

[10] B. Dabrowski, W. Swieszkowski, D. Godlinski, K. J. Kurzydlowski. Highly porous titanium scaffolds for orthopaedic applications. Journal of Biomedical Materials Research - Part B Applied Biomaterials 95(1):53-61, 2010. DOI:10.1002/jbm.b.31682

[11] D. Buser, R. K. Schenk, S. Steinemann, et al. Influence of surface characteristics on bone integration of titanium implants. A histomorphometric study in miniature pigs. Journal of Biomedical Materials Research 25(7):889-902, 1991. DOI:10.1002/jbm.820250708

[12] K. Gotfredsen, T. Berglundh, J. Lindhe. Anchorage of titanium implants with different surface characteristics: an experimental study in rabbits. Clinical implant dentistry and related research 2(3):120-128, 2000.

[13] A. Wennerberg, T. Albrektsson, B. Andersson, J. J. Krol. A histomorghometric study of screw-shaped and removal torque titanium implants with three different surface topographies. Clinical Oral Implants Research 6(1):24-30, 1995

DOI:10.1034/j.1600-0501.1995.060103.x
[14] F. Denk, F. Denk, A. Jíra. A dental implant shaft, 2017.

[15] M. I. Z. Ridzwan, S. Shuib, A. Y. Hassan, et al. Problem of stress shielding and improvement to the hip implant designs: A review, 2007. DOI:10.3923/jms.2007.460.467.

[16] J. Y. Rho, R. B. Ashman, C. H. Turner. Young's modulus of trabecular and cortical bone material: Ultrasonic and microtensile measurements. Journal of Biomechanics 26(2):111-119, 1993. DOI:10.1016/0021-9290(93)90042-D.

[17] M. Niinomi. Mechanical properties of biomedical titanium alloys. Materials Science and Engineering: A 243(1-2):231-236, 1998. DOI:10.1016/S0921-5093(97)00806-X

[18] S. C. Cowin, D. H. Hegedus. Bone remodeling II: theory of adaptive elasticity. Journal of Elasticity 6(3):313-326, 1976. 0016974131 DOI:10.1007/BF00041724

[19] W. Oliver, G. Pharr. An improved technique for determining hardness and elastic modulus using load and displacement sensing indentation experiments. Journal of Materials Research 7(6):1564-1583, 1992. DOI:10.1557/JMR.1992.1564 